\title{
The Effect of Cognitive Training in a Day Care Center in Patients with Early Alzheimer's Disease Dementia: A Retrospective Study
}

\author{
Hyuk Sung Kwon', Ha-rin Yang', Kyungtaek Yun², Jong Sook Baek², \\ Young Un Kim², Seongho Park ${ }^{3}$, and Hojin Choi ${ }^{1 凶}$ \\ ${ }^{1}$ Department of Neurology, Hanyang University College of Medicine, Seoul, Republic of Korea \\ ${ }^{2}$ Namyangju City Public Health Center, Namyangju, Republic of Korea \\ ${ }^{3}$ Department of Neurology, Inje University Haeundae Paik Hospital, Busan, Republic of Korea
}

Objective This study aimed to evaluate the effect of cognitive training programs on the progression of dementia in patients with early stage Alzheimer's disease dementia (ADD) at the day care center.

Methods From January 2015 to December 2018, a total of 119 patients with early ADD were evaluated. All subjects were classified into two groups according to participate in cognitive training program in addition to usual standard clinical care. Changes in scores for minimental status examination-dementia screening (MMSE-DS) and clinical dementia rating-sum of boxes (CDR-SOB) during the 12 months were compared between two groups. Multivariable logistic regression analyses were performed.

Results As compared to case-subjects ( $\mathrm{n}=43$ ), the MMSE-DS and CDR-SOB scores were significantly worse at 12 months in the control-subjects $(n=76)$. A statistically significant difference between the two groups was observed due to changes in MMSE-DS ( $p=0.012$ ) and CDR-SOB $(\mathrm{p}<0.001)$ scores. Multivariable logistic regression analysis showed that the cognitive training program (odds ratio and 95\% confidence interval: $0.225,0.070-0.725$ ) was independently associated with less progression of ADD.

Conclusion The cognitive training program was associated with benefits in maintaining cognitive function for patients with earlystage ADD that were receiving medical treatment.

Psychiatry Investig 2020;17(8):829-834

Key Words Cognitive training, Day care center, Alzheimer's disease.

\section{INTRODUCTION}

The population of South Korea is aging very rapidly due to the world's lowest birth rate and the extension of the average life expectancy in the country. The predicted life expectancy in South Korea will become the highest in the world, beyond Japan and France. ${ }^{1}$ Owing to the rapid aging, the number of dementia patients in South Korea exceeded 700,000 in 2017 and is expected to increase rapidly to 1 million in 2024 and 1.5 million in 2034. ${ }^{2}$ Thus, the burden of dementia patients and for their families will increase rapidly, and the social burden to manage them will also increase exponentially. In response, the

\footnotetext{
Received: May 12, 2020 Revised: June 12, 2020

Accepted: July 14, 2020

$\triangle$ Correspondence: Hojin Choi, MD, PhD

Department of Neurology, Hanyang University College of Medicine, Guri Hospital, 153 Gyeongchun-ro, Guri 11923, Republic of Korea

Tel: +82-31-560-2260, Fax: +82-31-560-2289, E-mail: chj@hanyang.ac.kr

(ac) This is an Open Access article distributed under the terms of the Creative Commons Attribution Non-Commercial License (https://creativecommons.org/licenses/bync/4.0) which permits unrestricted non-commercial use, distribution, and reproduction in any medium, provided the original work is properly cited.
}

Korean government has actively engaged in policy intervention on dementia management issues, ranging from the comprehensive plan announced by the Ministry of Health and Welfare in 2008 to the National Responsibility System for dementia announced in September 2017.,4

One of the major policies to reduce the burden of care for dementia patients and caregivers is the introduction of longterm care insurance. After the introduction of the special level (5th level) of long-term care insurance for dementia patients in 2014, this was expanded to include mild dementia patients who had not yet received benefits. ${ }^{5,6}$ This includes support for the use of day care centers and cognitive training programs. Since the implementation of the national responsibility for dementia, the benefit from long-term care insurance has been expanded to a wider range of early dementia patients in terms of cognitive support level. After this revision, most of the patients with early stage of dementia were able to use the day care center and cognitive training program. ${ }^{7,8}$

Day care centers for people with dementia is known to reduce the burden of caregivers. ${ }^{9}$ At this point, it is necessary to 
analyze how the use of cognitive training programs in day care centers helps to maintain and support the cognitive function of dementia patients in addition to reducing the burden for caregivers.

This study aimed to evaluate the effect of cognitive training programs on the progression of dementia in patients with early stage Alzheimer's disease dementia (ADD) at the day care center. It would give more information to determine the appropriate policy intervention for early dementia patients by examining the changes in cognitive function, according to the current status of participation in a cognitive training program.

\section{METHODS}

\section{Study population}

We retrospectively analyzed medical records from patients with $\mathrm{ADD}$ who were included in the local dementia mass screening program of the Namyangju City public health center between January 2015 and December 2018. This program was conducted for residents in the city who were aged 60 or older. The inclusion criteria of this study were: 1) diagnosed as clinically probable ADD by criteria of the National Institute of Neurologic and Communicative Disorders and Stroke-Alzheimer Disease and Related Disorders Association Standard (NINDS), ${ }^{10}$ 2) with mild (early-stage) symptoms [Clinical Dementia Rating Scale $(\mathrm{CDR}) \leq 1]{ }^{11} 3$ ) had received an acetylcholinesterase inhibitor, and 4) performed cognitive function tests 12 months after initial assessment.

Exclusion criteria were as follows: 1) any primary neurodegenerative or psychiatric disorder other than ADD (i.e., Parkinson's disease, schizophrenia, bipolar disorder or major depressive disorder), ${ }^{12,13} 2$ ) diagnosed as clinically possible, probable, or definite vascular dementia by criteria of the National Institute of Neurological Disorders and Stroke-the Association pour la Recherche et l'Enseignement en Neurosciences (NINDSAIREN), ${ }^{14}$ 3) brain tumor or severe traumatic brain injury, 4) any history of drug or alcohol addiction within the past 2 years, 5) cases of medical diseases (liver disease, kidney disease, or thyroid disease) that cause cognitive function decline, and 6) any physical disability (hearing or visual impairment) that could significantly impair the evaluation of the patient or cognitive training program.

All subjects were classified into two groups (Figure 1). The case-subjects had regularly visited the day care center (which is provided as a service from long-term care insurance), were taking the cognitive training program, and received the acetylcholinesterase inhibitor. The control-subjects continued to receive the acetylcholinesterase inhibitor without visiting day care center or taking cognitive training. All patients underwent physical and neurological examination. Demographic and clinical information including age, sex, education, vascular risk factor (hypertension, diabetes, dyslipidemia, smoking, and cardiovascular disease), and medication history were collected. The time point at which the patients initially underwent brain imaging (CT or MRI) and neuropsychological tests, including mini-mental status examination-dementia screening (MMSEDS), Clinical Dementia Rating (CDR), and Consortium to Establish a Registry for Alzheimer's Diseases (CERAD) by the local dementia mass screening program were considered to be the baseline.$^{15}$ Follow-up neuropsychological tests [MMSE-DS, $\mathrm{CDR}$, and sum-of-boxes (CDR-SOB)], which were conducted 12 months after baseline, were compared between the two groups. The MMSE-DS is a Korean version of the MMSE, which has been validated with standardized norms. ${ }^{16}$ We defined pro-

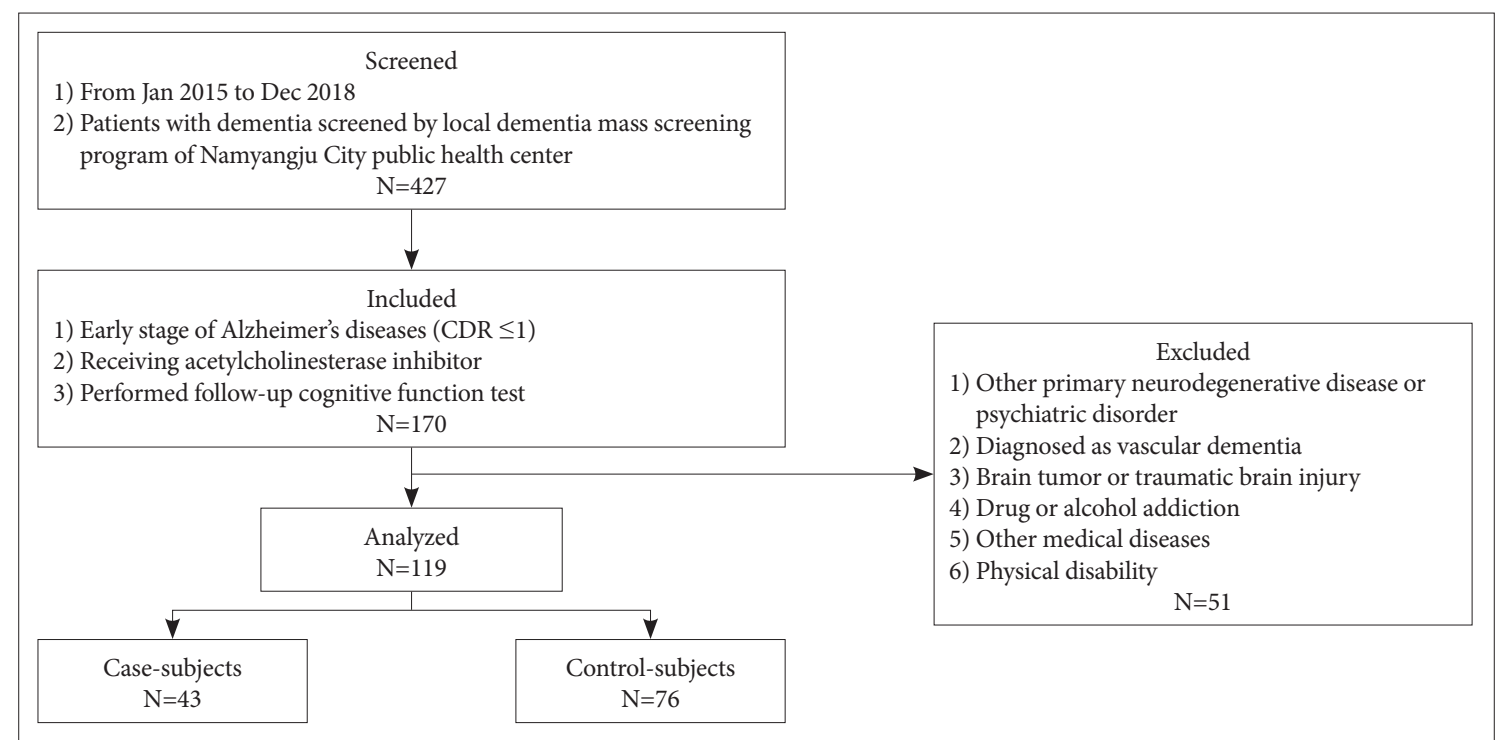

Figure 1. Patient enrollment. CDR: Clinical Dementia Rating. 
gression of $\mathrm{ADD}$ as an increase in $\mathrm{CDR}-\mathrm{SOB}$ score by $\geq 2$ points at 12 months. ${ }^{17}$ Informed consent was waived due to the retrospective nature of the study. This study was approved by the Institutional Review Board at the Hanyang University Guri Hospital (2020-03-008).

\section{Cognitive training in the day care center}

The cognitive training program was designed and assessed using standard guidelines for coordinating dementia support center. The cognitive training was administered 3 hours in a day, 5 days a week, and for 12 months at each day care center. There was an instructor at each of day care center, who was an experienced clinical neuropsychologist. Programs included attention training (i.e., paper or computer assisted attention training), memory training (i.e., training in the recall of a list and remembering the location of objects in the room), visuoconstruction training (i.e., drawing various things and changing blocks), physical training (i.e., massed calisthenics), occupational training (i.e., creative activity such as drawing or knitting), and speech training.

\section{Statistics}

We performed Fisher's exact tests to compare proportions and the Mann-Whitney U test and Student's t-test for continuous variables to evaluate differences between the groups. Changes in cognitive assessment scores (MMSE-DS and CDR-SOB scores) between baseline and the follow-up point were analyzed by Wilcoxon signed-rank test. The scores of the two groups for baseline and follow-up points, were compared using MannWhitney. Correlation between ADD progression and patient characteristics were evaluated by logistic regression analysis. Adjusted variables were age, sex, and factors which were significant ( $p$ value $<0.1$ ) in the univariate analysis. Two-tailed $p$ values less than 0.05 were considered significant, and all statistical analyses were performed using SPSS 21.0 package for Windows (IBM Corp., Armonk, NY, USA).

\section{RESULTS}

Overall, 427 dementia patients were screened by the local dementia mass screening program of the Namyangju City public health center between January 2015 and December 2018. Among them, 170 patients were diagnosed as early-stage ADD $(\mathrm{CDR} \leq 1)$; patients underwent follow-up neurocognitive function tests, and received acetylcholinesterase inhibitors. After excluding patients who met the exclusion criteria, a total of 119 patients were included in this study (Figure 1). Patient ages ranged from 67 to 89 years [mean $( \pm \mathrm{SD})$ was $78.0( \pm 4.4)$ years].

The number of patients who received both cognitive training and standard clinical care (case-subjects) was 43 and the num- ber of patients who received only standard clinical care (control-subjects) was 76. The baseline characteristics of each group are shown in Table 1. Progression of ADD and dyslipidemia were identified more frequently in control-subjects and baseline CDR-SOB score was higher in case-subjects. Otherwise, there were no significant differences between the two groups.

As compared to case-subjects, the MMSE-DS and CDR-SOB scores significantly worsened at 12 months in the control-subjects (Table 2, Figure 2). The mean increase in the MMSE-DS scores in case-subjects (treated with both cognitive training and medication) during the 12 months was 0.45 points ( $\mathrm{p}=0.248$ vs. baseline), whereas the mean decrease in the MMSE-DS score in the control-subjects (treated with medication only) was 1.80 points ( $p<0.001$ vs. baseline). The mean increase of the CDRSOB score was 0.64 points ( $\mathrm{p}=0.080$ vs. baseline) in case-subjects, whereas the mean increase in CDR-SOB score was 2.82 points ( $\mathrm{p}<0.001$ vs. baseline) in control-subjects. A statistically significant difference between the two groups was observed in the changes in MMSE-DS and CDR-SOB scores (Figure 2).

Patient characteristics based on progression of dementia are shown in Table 3. Patients with progression of dementia had

Table 1. Clinical characteristics of case-subjects and controlsubjects

\begin{tabular}{lccc}
\hline & $\begin{array}{c}\text { Case-subjects } \\
(\mathrm{N}=43)\end{array}$ & $\begin{array}{c}\text { Control- } \\
\text { subjects } \\
(\mathrm{N}=76)\end{array}$ & p value* \\
\hline Age, mean \pm SD (years) & $77.5 \pm 4.4$ & $78.3 \pm 4.4$ & $0.323^{\dagger}$ \\
Sex, female & $31(72.1)$ & $55(72.4)$ & 0.974 \\
Medical history & & & \\
$\quad$ Hypertension & $19(44.2)$ & $40(52.6)$ & 0.376 \\
$\quad$ Diabetes mellitus & $16(37.2)$ & $32(42.1)$ & 0.601 \\
$\quad$ Dyslipidemia & $11(25.6)$ & $35(46.1)$ & 0.028 \\
$\quad$ Cardiovascular disease & $12(27.9)$ & $19(25.3)$ & 0.760 \\
$\quad$ Current smoker & $11(25.6)$ & $20(26.3)$ & 0.930 \\
Education, year & $5.0 \pm 4.2$ & $5.2 \pm 4.3$ & $0.819^{\dagger}$ \\
Live alone & $5(11.6)$ & $12(15.8)$ & 0.533 \\
Family history of dementia & $12(27.9)$ & $15(19.7)$ & 0.307 \\
Baseline MMSE-DS score & $15.4 \pm 4.9$ & $15.5 \pm 4.5$ & $0.989^{\ddagger}$ \\
Follow up MMSE-DS score & $16.1 \pm 6.0$ & $13.7 \pm 3.8$ & $0.036^{\ddagger}$ \\
Baseline CDR-SOB score & $4.9 \pm 0.7$ & $5.3 \pm 0.7$ & $0.012^{\ddagger}$ \\
Follow up CDR-SOB score & $5.6 \pm 2.1$ & $8.0 \pm 3.0$ & $<0.001^{\ddagger}$ \\
Progression of ADD & $4(9.3)$ & $27(35.5)$ & 0.002 \\
\hline Data are presented a number & &
\end{tabular}

Data are presented as number of patients (\%) unless otherwise indicated. * p value for comparison between case-subjects and control-subjects, ${ }^{\dagger}$ student’s t-test, ${ }^{\ddagger}$ Mann-Whitney U test, and Fisher’s exact test were used as appropriate. SD: standard deviation, MMSE-DS: mini-mental status examination-dementia screening, CDR-SOB: Clinical Dementia Rating Scale sum of boxes, ADD: Alzheimer's disease dementia 
Table 2. Evaluation of the cognition function in case-subjects and control-subjects during 12 months

\begin{tabular}{|c|c|c|c|c|c|c|}
\hline & \multicolumn{3}{|c|}{ Case-subjects } & \multicolumn{3}{|c|}{ Control-subjects } \\
\hline & Baseline & Follow up & $\mathrm{p}$ & Baseline & Follow up & $\mathrm{p}$ \\
\hline MMSE-DS score & $15.4 \pm 4.9$ & $16.1 \pm 6.0$ & 0.248 & $15.5 \pm 4.5$ & $13.7 \pm 3.8$ & $<0.001$ \\
\hline CDR-SOB score & $4.9 \pm 0.7$ & $5.6 \pm 2.1$ & 0.080 & $5.3 \pm 0.7$ & $8.0 \pm 3.0$ & $<0.001$ \\
\hline
\end{tabular}

p-value for comparison between baseline and follow up by Wilcoxon signed-rank test. MMSE-DS: mini-mental status examination-dementia screening, CDR-SOB: Clinical Dementia Rating Scale sum of boxes
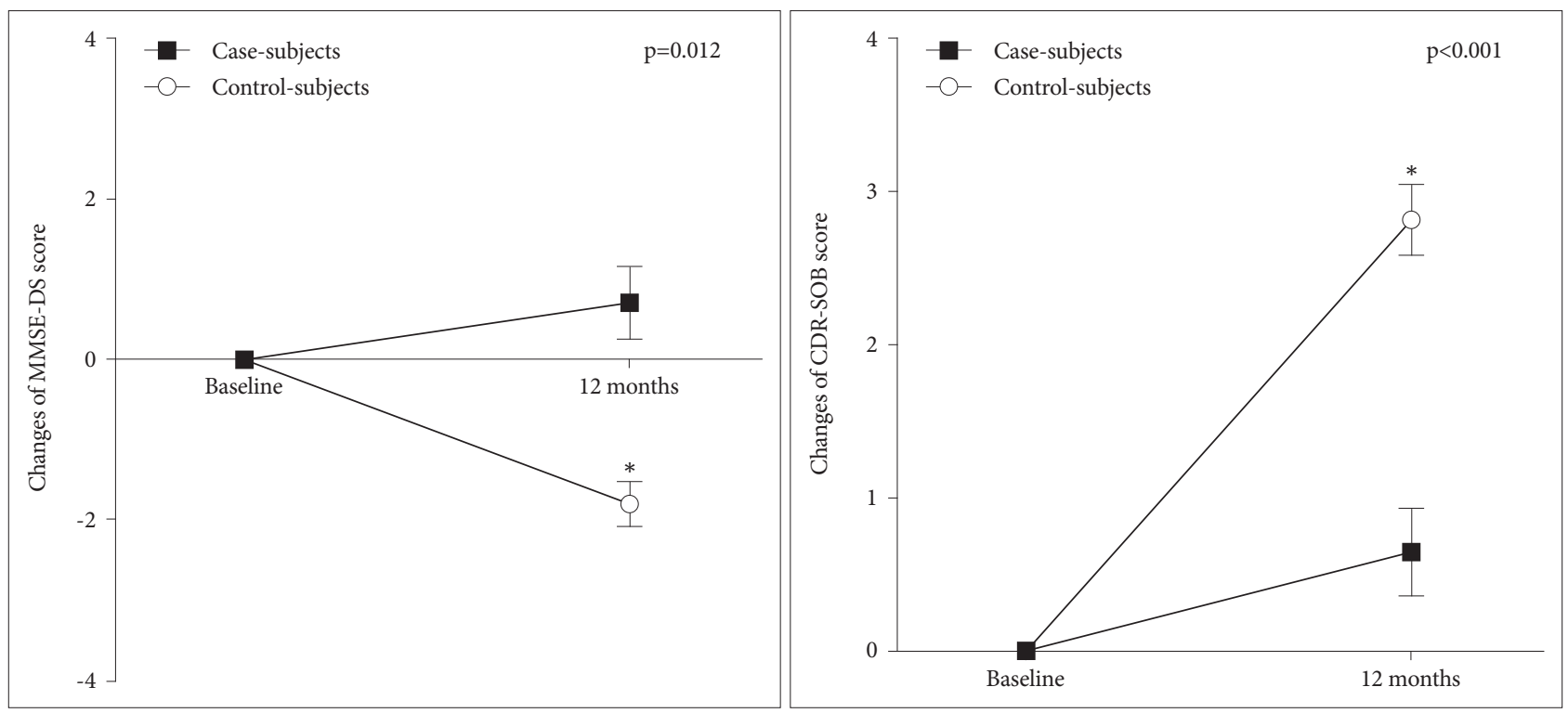

Figure 2. MMSE-DS score changes and CDR-SOB scores in case and control subjects during the follow-up period. Note the significant preservation of MMSE-DS $(p=0.012)$ and the CDR-SOB score $(p<0.001)$ at 12 months in case subjects compared to the decrease in control subjects. Data are the means \pm SEM. Indicated $p$-value represents the difference in score change between two groups. ${ }^{*} p<0.001$ versus baseline. MMSE-DS: mini-mental status examination-dementia screening, CDR-SOB: Clinical Dementia Rating Scale sum of boxes.

more dyslipidemia $(\mathrm{p}=0.011)$ and less cognitive training $(\mathrm{p}=$ 0.002). Logistic regression analysis adjusted for age, sex, and p-value below 0.1 on univariate analysis (dyslipidemia, education, and cognitive training) showed additional cognitive training (odds ratio and 95\% confidence interval: 0.225, 0.070-0.725) were independently associated with less progression of ADD (Table 4).

\section{DISCUSSION}

In this study, application of a cognitive training program at a day care center in addition to usual standard clinical care showed significant benefit in terms of cognitive function in early ADD patients. In the management of ADD, pharmacological therapy helps to suppress the progression of symptoms, but it does not fundamentally change the progression of the disease. Accordingly, various non- pharmacological therapies have been attempted, including cognitive training programs, which have been identified as the most important non-pharmacological approach. ${ }^{18-23}$ The results of our study demonstrated that cognitive function was maintained and that deterioration associ- ated with the disease was also suppressed significantly in the group that continued to perform cognitive function training, even in patients who had been treated consistently with medication. This finding is consistent with previous studies that cognitive training can be effective in improving cognitive function and activities of daily living in dementia patients. ${ }^{24-28}$ However, previous studies have primarily observed patients for a shortterm period, and under intensive training program conditions. Therefore, the findings for comparison are limited and the impact of public services, such the provision of cognitive training programs by the long-term care insurance, are still being investigated. In particular, similar to our study, a study investigated the change in cognitive function, based on the provision of cognitive function programs by the grade of long-term care insurance in South Korea; however, the results were challenging to directly apply in the clinical field or for dementia-related policy because the clinical condition of the patients was not fully considered. ${ }^{6}$ Therefore, our study is significant because it evaluated patient participation in a cognitive training program and changes in clinical symptoms, including cognitive function, which can be accurately tracked through medical records. In 
addition, the patient's diagnosis and clinical severity can be accurately identified through comprehensive examination, including the cognitive function test and brain imaging that are conducted by a dementia specialist.

Dyslipidemia was independently associated with cognitive decline in this study. However it is difficult to interpret the meaning of this result in this study, owing to the small sample size and insufficient data for compliance with medications. Although there is a possibility for a correlation between dyslipidemia and cognitive decline in specific patients, ${ }^{29,30}$ the association between

Table 3. Clinical characteristics of according to progression of ADD

\begin{tabular}{lrrr}
\hline & $\begin{array}{c}\text { Not progressed } \\
(\mathrm{N}=88)\end{array}$ & $\begin{array}{c}\text { Progressed } \\
(\mathrm{N}=31)\end{array}$ & p value* \\
\hline Age, mean \pm SD (years) & $77.7 \pm 4.0$ & $79.0 \pm 5.3$ & $0.142^{\dagger}$ \\
Sex, female & $60(74.1)$ & $26(68.4)$ & 0.521 \\
Medical history & & & \\
$\quad$ Hypertension & $37(45.7)$ & $22(57.9)$ & 0.214 \\
$\quad$ Diabetes mellitus & $36(44.4)$ & $12(31.6)$ & 0.182 \\
$\quad$ Dyslipidemia & $25(30.9)$ & $21(55.3)$ & 0.011 \\
$\quad$ Cardiovascular disease & $23(28.8)$ & $8(21.1)$ & 0.375 \\
$\quad$ Current smoker & $18(22.2)$ & $13(34.2)$ & 0.165 \\
Education, year & $4.7 \pm 4.0$ & $6.4 \pm 4.7$ & $0.058^{\dagger}$ \\
Live alone & $12(13.6)$ & $5(16.1)$ & 0.733 \\
Family history of dementia & $19(23.5)$ & $8(21.1)$ & 0.770 \\
Baseline MMSE-DS score & $15.4 \pm 4.3$ & $15.8 \pm 5.6$ & $0.119^{\ddagger}$ \\
Follow up MMSE-DS score & $15.3 \pm 4.7$ & $12.4 \pm 4.6$ & $0.025^{\ddagger}$ \\
Baseline CDR-SOB score & $5.1 \pm 0.7$ & $5.2 \pm 0.9$ & $0.140^{\ddagger}$ \\
Follow up CDR-SOB score & $5.6 \pm 1.0$ & $11.7 \pm 1.8$ & $<0.001^{\ddagger}$ \\
Receive cognitive training & $39(44.3)$ & $4(12.9)$ & 0.002 \\
\hline
\end{tabular}

Data are presented as number of patients (\%) unless otherwise indicated. Progression of ADD was defined as increase in CDR-SOB score by $\geq 2$ points at 12 months. ${ }^{*}$ p value for comparison between case-subjects and control-subjects, †'student's t-test, ‡Mann-Whitney $\mathrm{U}$ test, and Fisher's exact test were used as appropriate. SD: standard deviation, ADD: Alzheimer's disease dementia, MMSEDS: mini-mental status examination-dementia screening, CDRSOB: Clinical Dementia Rating-sum of boxes dyslipidemia and ADD is still controversial and requires additional research. ${ }^{31}$

There were several limitations to this study. First, it was retrospectively designed, and has the possibility of significant selection bias. Therefore, we applied strict inclusion and exclusion criteria based on the medical records to try to reduce this bias. Second, as the time of enrollment was different for each participants, received cognitive training program might be different among them. However contents of the cognitive training programs are structured and do not make a big difference every year. Third, attendance and compliance rate for cognitive training was not monitored. It would be difficult for some participants to attend three hours of cognitive training for five days a week. But, it is also meaningful that application of a cognitive training program showed benefit although some of them might have low attendance rate. Fourth, biomarkers including magnetic resonance imaging, positron emission tomography imaging, and cerebrospinal fluid study were not analyzed in this study. Fifth, lifestyle risk factors and burden of caregivers are not investigated. Finally, the sample size was small, therefore a study with a prospective design, standardized diagnostic workup including biomarkers, investigating life style risk factors and burden of caregivers, and larger number of participants may be warranted. However, considering the advantages of this study, the results are sufficiently suggestive for clinical sites.

Another factor to consider is that more than half of the early dementia patients who are constantly receiving medication do not receive cognitive training programs at their day care center. Therefore, it is necessary to consider not only the provision of the cognitive training programs at day care centers through long-term care insurance, but also ways to actively provide these programs throughout the medical field to make them more accessible for patients. In conclusion, the provision of cognitive training programs through long-term care insurance at day care centers have possibility to help maintain the cognitive function in early-stage ADD patients. Based on these results, these programs should be expanded in the future, and policies should consider the way to utilize these programs in the medical field.

Table 4. Multivariable logistic regression analysis of factors for progression of ADD

\begin{tabular}{lccc}
\hline & Unadjusted OR & Adjusted OR & p-value \\
\hline Cognitive training & $0.186(0.060-0.577)$ & $0.225(0.070-0.725)$ & 0.012 \\
Age & $1.073(0.976-1.180)$ & $1.077(0.968-1.193)$ & 0.131 \\
Sex, female & $0.917(0.370-2.269)$ & $1.730(0.580-5.161)$ & 0.325 \\
Education, per 1 year increase & $1.097(0.996-1.210)$ & $1.112(0.998-1.239)$ & 0.055 \\
Dyslipidemia & $2.967(1.277-6.891)$ & $2.572(1.013-6.530)$ & 0.047 \\
\hline
\end{tabular}

p-value for multivariate models. Data are presented as odds ratios (OR; 95\% confidence interval). Adjusted for age, sex, cognitive training, education, and dyslipidemia. Progression of ADD was defined as increase in CDR-SOB score by $\geq 2$ points at 12 months. ADD: Alzheimer's disease dementia, CDR-SOB: Clinical Dementia Rating Scale sum of boxes 


\section{Acknowledgments}

This work was supported by the research fund of Hanyang University (HY-2017).

\section{Conflicts of Interest}

The authors have no potential conflicts of interest to disclose.

\section{Author Contributions}

Conceptualization: Hyuk Sung Kwon, Hojin Choi. Data curation: Kyungtaek Yun, Jong Sook Baek, Young Un Kim. Formal analysis: Hyuk Sung Kwon, Seongho Park. Funding acquisition: Hojin Choi. Investigation: Hyuk Sung Kwon, Hojin Choi. Methodology: Hyuk Sung Kwon, Ha-rin Yang. Project administration: Hojin Choi. Supervision: Ha-rin Yang, Kyungtaek Yun, Jong Sook Baek, Young Un Kim. Validation: Hyuk Sung Kwon, Harin Yang. Visualization: Hyuk Sung Kwon. Writing_original draft: Hyuk Sung Kwon. Writing_-review \& editing: Seongho Park, Hojin Choi.

\section{ORCID iDs}

$\begin{array}{ll}\text { Hyuk Sung Kwon } & \text { https://orcid.org/0000-0002-2005-0983 } \\ \text { Ha-rin Yang } & \text { https://orcid.org/0000-0001-7319-6890 } \\ \text { Kyungtaek Yun } & \text { https://orcid.org/0000-0002-1485-079X } \\ \text { Jong Sook Baek } & \text { https://orcid.org/0000-0002-0772-3784 } \\ \text { Young Un Kim } & \text { https://orcid.org/0000-0003-0743-9852 } \\ \text { Seongho Park } & \text { https://orcid.org/0000-0002-0504-2539 } \\ \text { Hojin Choi } & \text { https://orcid.org/0000-0002-9637-4423 }\end{array}$

\section{REFERENCES}

1. Kontis V, Bennett JE, Mathers CD, Li G, Foreman K, Ezzati M. Future life expectancy in 35 industrialised countries: projections with a Bayesian model ensemble. Lancet 2017;389:1323-1335.

2. National Institute of Dementia. 2017 National Institute of Dementia Annual Report. Available at: https://www.nid.or.kr/info/dataroom view.aspx?bid=168. Accessed May 12, 2020.

3. Choi H, Kim SH. Policy of national responsibility and dementia care. J Korean Med Assoc 2018;61:309-313.

4. Lee DW, Seong SJ. Korean national dementia plans: from 1st to 3rd. J Korean Med Assoc 2018;61:298-303.

5. Lee YK. The introduction of special level for people with dementia in the eligibility criteria of long-term care insurance system in Korea. Korea Institute for Health and Social Affairs (KIHASA) Health and Welfare Issue and Focus 2014;245:1-8.

6. Ju YJ, Nam CM, Lee SG, Park S, Hahm MI, Park EC. Evaluation of the South Korean national long-term care insurance-funded cognitive function training programme for older people with mild dementia. Age Ageing 2019;48:636-642.

7. Kim YI. Manual for person centered cognitive support service. National Health Insurance Service, 2019 July. Available at: http://www. longtermcare.or.kr/npbs/e/b/102/npeb102m01.web?menuId=npe0000 000040\&zoomSize $=$. Accessed May 12, 2020.

8. Choi H, Kim SH, Lee J-H, Lee AY, Park KW, Lee EA, et al. National responsibility policy for dementia care: current and future. J Korean Neurol Assoc 2018;36:152-158

9. Tretteteig S, Vatne S, Rokstad AM. The influence of day care centres designed for people with dementia on family caregivers - a qualitative study. BMC Geriatr 2017;17:5.

10. McKhann G, Drachman D, Folstein M, Katzman R, Price D, Stadlan EM. Clinical diagnosis of Alzheimer's disease: report of the NINCDSADRDA work group under the auspices of department of health and human services task force on Alzheimer's disease. Neurology 1984; 34:939-944.

11. Morris JC. The Clinical Dementia Rating (CDR): current version and scoring rules. Neurology 1993;43:2412-2414.

12. Cho SJ, Kim J, Kang YJ, Lee SY, Seo HY, Park JE, et al. Annual preva- lence and incidence of schizophrenia and similar psychotic disorders in the Republic of Korea: a national health insurance data-based study. Psychiatry Investig 2020;17:61-70.

13. Muneer A, Mazommil R. The staging of major mood disorders: clinical and neurobiological correlates. Psychiatry Investig 2018;15:747-758.

14. Roman GC, Tatemichi TK, Erkinjuntti T, Cummings JL, Masdeu JC, Garcia JH, et al. Vascular dementia: diagnostic criteria for research studies. Report of the NINDS-AIREN International Workshop. Neurology 1993;43:250-260.

15. Lee JH, Lee KU, Lee DY, Kim KW, Jhoo JH, Kim JH, et al. Development of the Korean version of the Consortium to Establish a Registry for Alzheimer's Disease Assessment Packet (CERAD-K): clinical and neuropsychological assessment batteries. J Gerontol B Psychol Sci Soc Sci 2002;57:P47-P53.

16. Kim TH, Jhoo JH, Park JH, Kim JL, Ryu SH, Moon SW, et al. Korean version of mini mental status examination for dementia screening and its' short form. Psychiatry Investig 2010;7:102-108.

17. Williams MM, Storandt M, Roe CM, Morris JC. Progression of Alzheimer's disease as measured by Clinical Dementia Rating Sum of Boxes scores. Alzheimers Dement 2013;9:S39-S44.

18. Talassi E, Guerreschi M, Feriani M, Fedi V, Bianchetti A, Trabucchi M. Effectiveness of a cognitive rehabilitation program in mild dementia (MD) and mild cognitive impairment (MCI): a case control study. Arch Gerontol Geriatr 2007;44(Suppl 1):391-399.

19. Langenbahn DM, Ashman T, Cantor J, Trott C. An evidence-based review of cognitive rehabilitation in medical conditions affecting cognitive function. Arch Phys Med Rehabil 2013;94:271-286.

20. Brueggen K, Kasper E, Ochmann S, Pfaff H, Webel S, Schneider W, et al. Cognitive rehabilitation in Alzheimer's disease: a controlled intervention trial. J Alzheimers Dis 2017;57:1315-1324.

21. Bahar-Fuchs A, Clare L, Woods B. Cognitive training and cognitive rehabilitation for persons with mild to moderate dementia of the Alzheimer's or vascular type: a review. Alzheimers Res Ther 2013;5:35.

22. Choi J, Twamley EW. Cognitive rehabilitation therapies for Alzheimer's disease: a review of methods to improve treatment engagement and self-efficacy. Neuropsychol Rev 2013;23:48-62.

23. Kang MJ, Kim SM, Han SE, Bae JH, Yu WJ, Park MY, et al. Effect of paper-based cognitive training in early stage of Alzheimer's dementia. Dement Neurocogn Disord 2019;18:62-68.

24. Rebok GW, Ball K, Guey LT, Jones RN, Kim HY, King JW, et al. Tenyear effects of the advanced cognitive training for independent and vital elderly cognitive training trial on cognition and everyday functioning in older adults. J Am Geriatr Soc 2014;62:16-24.

25. Barban F, Annicchiarico R, Pantelopoulos S, Federici A, Perri R, Fadda $\mathrm{L}$, et al. Protecting cognition from aging and Alzheimer's disease: a computerized cognitive training combined with reminiscence therapy. Int J Geriatr Psychiatry 2016;31:340-348.

26. Fernandez-Calvo B, Contador I, Ramos F, Olazaran J, Mograbi DC, Morris RG. Effect of unawareness on rehabilitation outcome in a randomised controlled trial of multicomponent intervention for patients with mild Alzheimer's disease. Neuropsychol Rehabil 2015;25:448-477.

27. Buschert V, Bokde AL, Hampel H. Cognitive intervention in Alzheimer disease. Nat Rev Neurol 2010;6:508-517.

28. Na R, Yang JH, Yeom Y, Kim YJ, Byun S, Kim K, et al. A systematic review and meta-analysis of nonpharmacological interventions for moderate to severe dementia. Psychiatry Investig 2019;16:325-335.

29. Noori H, Gheini MR, Rezaeimanesh N, Saeedi R, Rezaei Aliabadi H, Sahraian MA, et al. The correlation between dyslipidemia and cognitive impairment in multiple sclerosis patients. Mult Scler Relat Disord 2019;36:101415.

30. Carlsson CM. Type 2 diabetes mellitus, dyslipidemia, and Alzheimer's disease. J Alzheimers Dis 2010;20:711-722.

31. Reitz C. Dyslipidemia and the risk of Alzheimer's disease. Curr Atheroscler Rep 2013;15:307. 